Supporting Information for

\title{
Functionalized Spiro- and Fused-Ring Heterocycles via Oxidative Demetalation of Cyclohexadienyl Ruthenium Complexes
}

\author{
F. Christopher Pigge, * John J. Coniglio, and Nigam P. Rath
}

TABLE OF CONTENTS

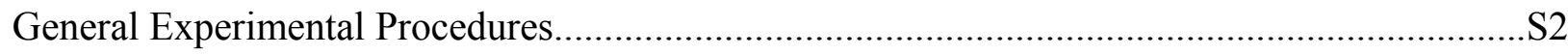

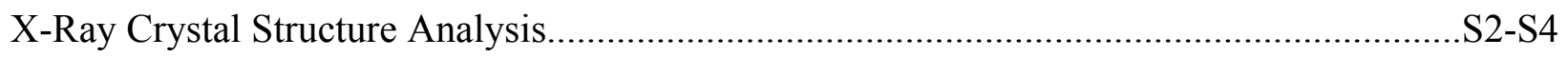

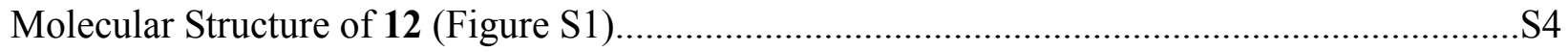

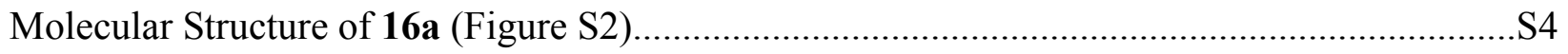

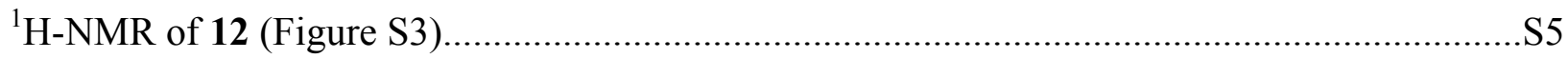

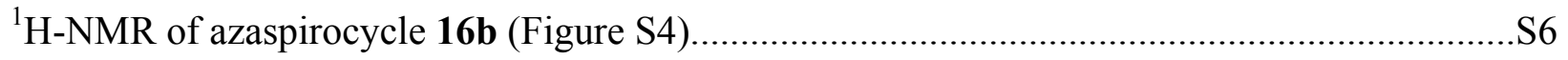

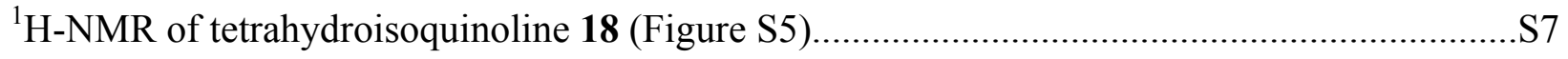

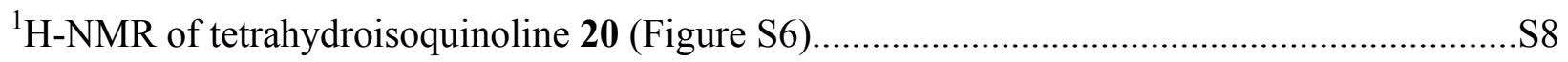

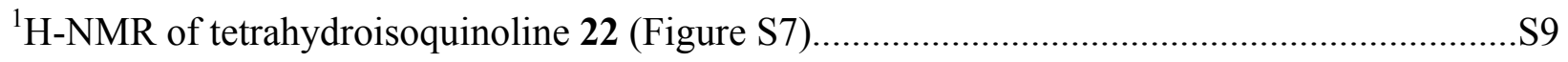

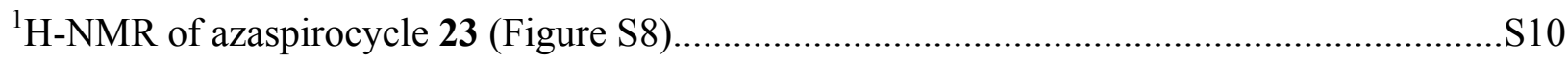

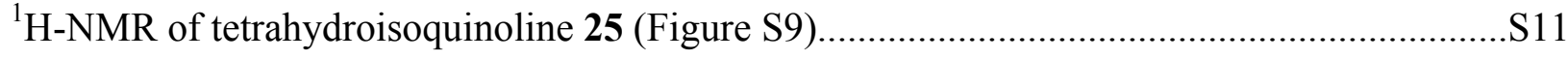

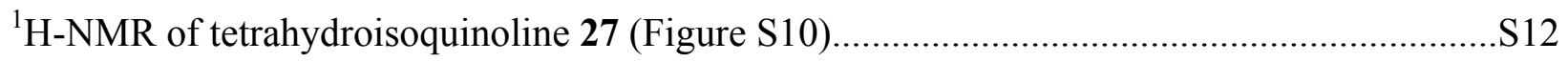

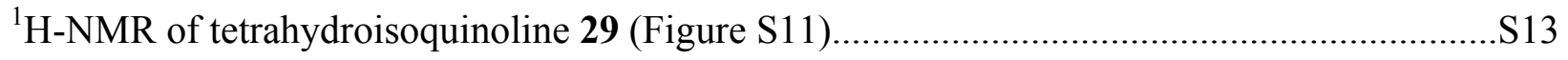

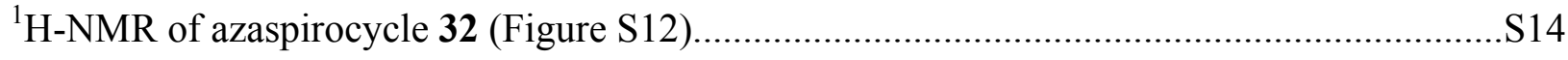

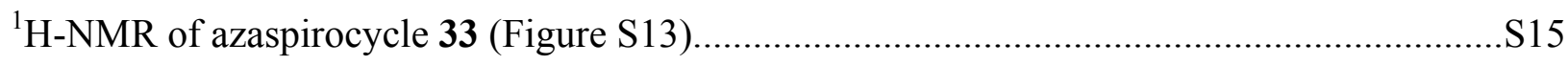

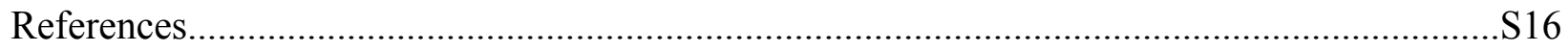


General Experimental. All commercially available reagents and solvents were used as received unless otherwise noted. Tetrahydrofuran was distilled from $\mathrm{Na}$-benzophenone, $\mathrm{CH}_{2} \mathrm{Cl}_{2}$ was distilled from $\mathrm{CaH}_{2}$. All reactions were performed in oven-dried glassware under a blanket of dry argon. Thin-layer chromatography was performed on silica gel 60 glass-backed TLC plates $(250 \mu \mathrm{m})$. All reactions were monitored by TLC for consumption of starting substrate. Flash column chromatography was performed using silica gel 60 (200-400 mesh). ${ }^{1} \mathrm{H}$ - and ${ }^{13} \mathrm{C}-\mathrm{NMR}$ spectra were obtained at 300 or $500 \mathrm{MHz}$ as indicated. Chemical shifts $(\delta)$ are reported relative to residual solvent peaks. IR spectra were recorded on an FT-IR spectrophotometer equipped with an ATR attachment. Melting points were determined using a capillary melting point apparatus and are uncorrected. High-resolution mass spectra were obtained using electron impact (EI) or positive ion FAB ionization. All cyclohexadienyl ruthenium complexes of the type 2 were prepared according to previously described procedures. ${ }^{1}$

X-Ray Crystal Structure Determination of 12 and 16a. Single crystals with dimensions 0.34 x $0.20 \times 0.10 \mathrm{~mm}(\mathbf{1 2})$ and $0.24 \times 0.22 \times 0.20 \mathrm{~mm}(\mathbf{1 6 a})$ were mounted on glass fibers in random orientation. Preliminary examination and data collection was performed using a Charge Coupled Device (CCD) Detector single crystal X-Ray diffractometer using graphite monochromated Mo K $\alpha$ radiation $(\lambda=0.71073 \AA$ ) equipped with a sealed tube X-Ray source. Preliminary unit cell constants were determined with a set of 45 narrow frames $\left(0.3^{\circ}\right.$ in $\left.\varpi\right)$ scans. Data collection consisted of 3636 frames of intensity data collected with a frame width of $0.3^{\circ}$ in $\varpi$ and counting time of $15 \mathrm{~seconds} /$ frame at a crystal to detector distance of $4.950 \mathrm{~cm}$. The double pass method of scanning was used to exclude any noise. The collected frames were integrated using an orientation matrix determined from the narrow frame scans. SMART and SAINT software 
packages were used for data collection and data integration. ${ }^{2}$ Analysis of the integrated data did not show any decay. Final cell constants were determined by global refinement of xyz centroids of threshold reflections from the complete data set. Collected data were corrected for systematic errors using SADABS based on the Laue symmetry using equivalent reflections. ${ }^{3}$

The structures were solved and refined using the SHELXTL- PLUS software package. ${ }^{4}$ Structures were solved by direct methods and refined successfully in the monclinic space groups $\mathrm{P} 2{ }_{1} / \mathrm{c}$ (12) and $\mathrm{P} 2_{1}$ (16a). Full matrix least-squares refinement was performed by minimizing $\Sigma \mathrm{w}\left(\mathrm{F}_{\mathrm{o}}{ }^{2}-\mathrm{F}_{\mathrm{c}}{ }^{2}\right)^{2}$. The non-hydrogen atoms were refined anisotropically to convergence. The hydrogen atoms were treated using appropriate riding model (AFIX m3). Complete crystallographic details will be deposited with the Cambridge Crystallographic Database.

Crystal data: $12-\mathrm{C}_{12} \mathrm{H}_{15} \mathrm{NO}_{3}, \mathrm{M}=221.25$, monoclinic, space group $\mathrm{P} 2{ }_{1} / \mathrm{c}, a=10.4813(6), b$ $=11.9342(7), c=9.7556(5) \AA, \beta=106.066(4)^{\circ}, \mathrm{V}=1172.63(11) \AA^{3}, \mathrm{Z}=4, \mathrm{D}_{\text {calc }}=1.253 \mathrm{~g} \mathrm{~cm}^{-3}$, $\mathrm{T}=180(2) \mathrm{K}, \mu(\mathrm{Mo}-\mathrm{K} \alpha)=0.090 \mathrm{~mm}^{-1}, 27565$ reflections collected of which 2094 were independent $\left(\mathrm{R}_{\text {int }}=0.11\right)$, all non-hydrogen atoms were refined anisotropically using full matrix least squares on $\mathrm{F}^{2}$ to give $\mathrm{R}_{1}=0.0751$ and $\mathrm{wR}_{2}=0.1975$.

Crystal data: $16 a-\mathrm{C}_{15} \mathrm{H}_{19} \mathrm{NO}_{4}, \mathrm{M}=221.25$, monoclinic, space group $\mathrm{P}_{1}, a=9.0063(2), b=$ 7.1259(1), $c=11.4408(2) \AA, \beta=110.754(1)^{\circ}, \mathrm{V}=686.60(2) \AA^{3}, \mathrm{Z}=2, \mathrm{D}_{\text {calc }}=1.341 \mathrm{~g} \mathrm{~cm}^{-3}, \mathrm{~T}=$ $200(2) \mathrm{K}, \mu(\mathrm{Mo}-\mathrm{K} \alpha)=0.097 \mathrm{~mm}^{-1}, 14701$ reflections collected of which 1789 were independent $\left(\mathrm{R}_{\text {int }}=0.03\right)$, all non-hydrogen atoms were refined anisotropically using full matrix least squares on $\mathrm{F}^{2}$ to give $\mathrm{R}_{1}=0.0324$ and $\mathrm{wR}_{2}=0.0861$. 
Figure S1. Molecular structure of 12 (ORTEP).

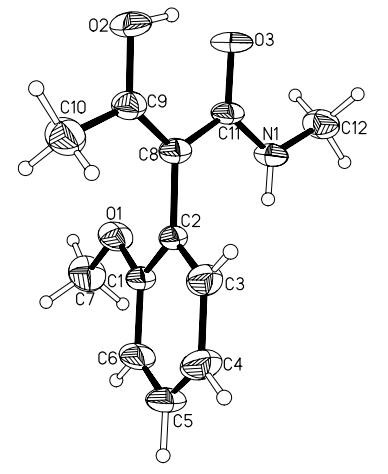

Figure S2. Molecular structure of 16a (ORTEP).

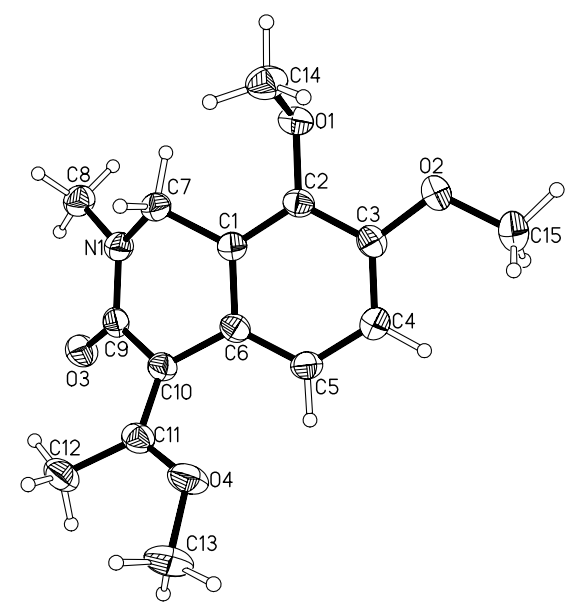




\section{Figure S3}
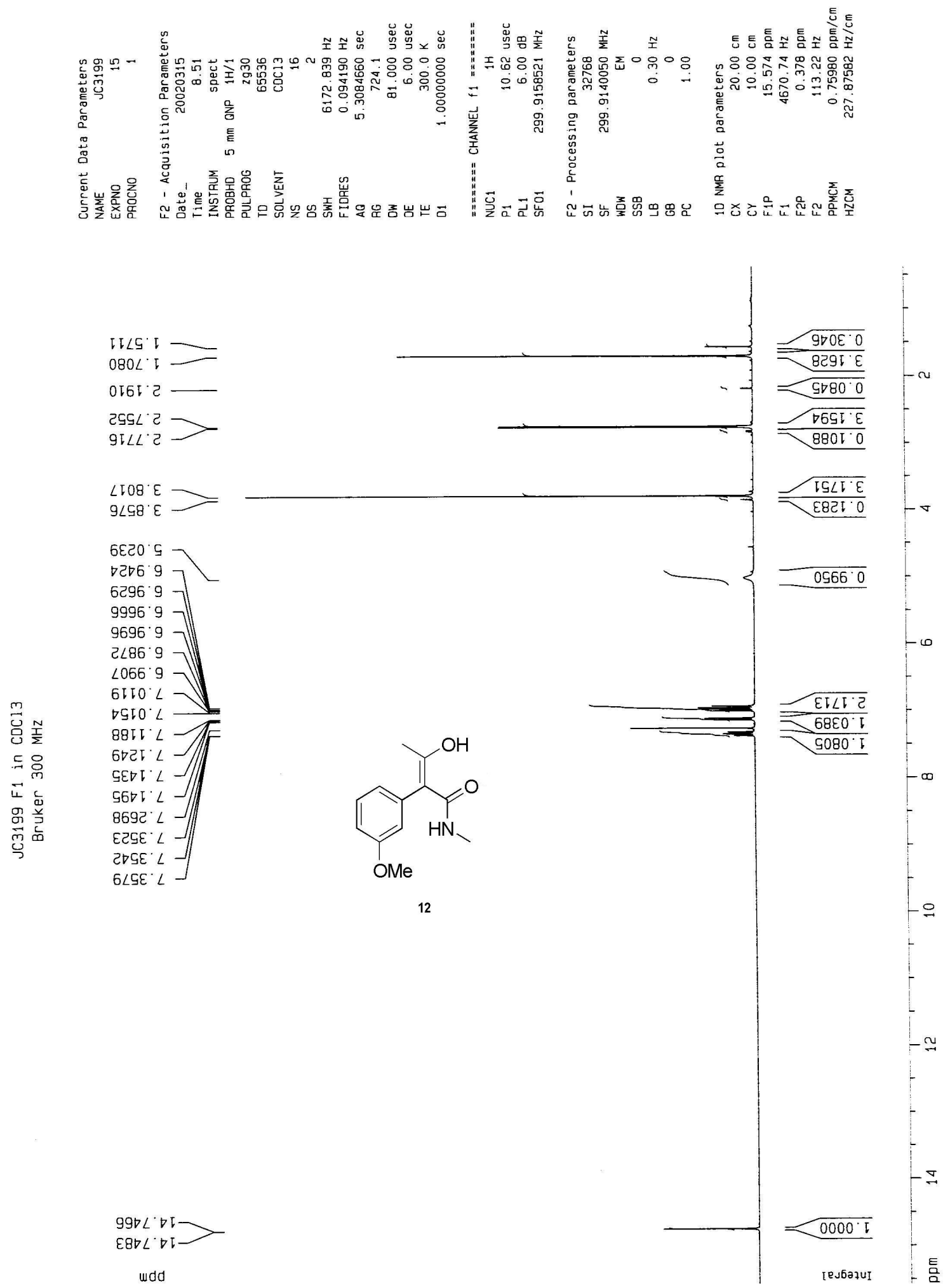


\section{Figure S4}

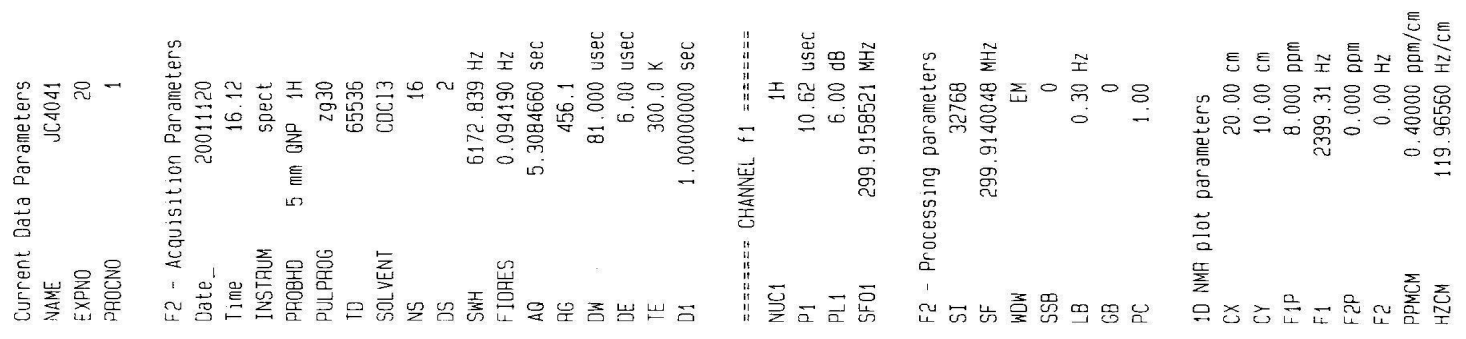

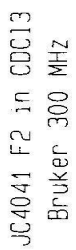

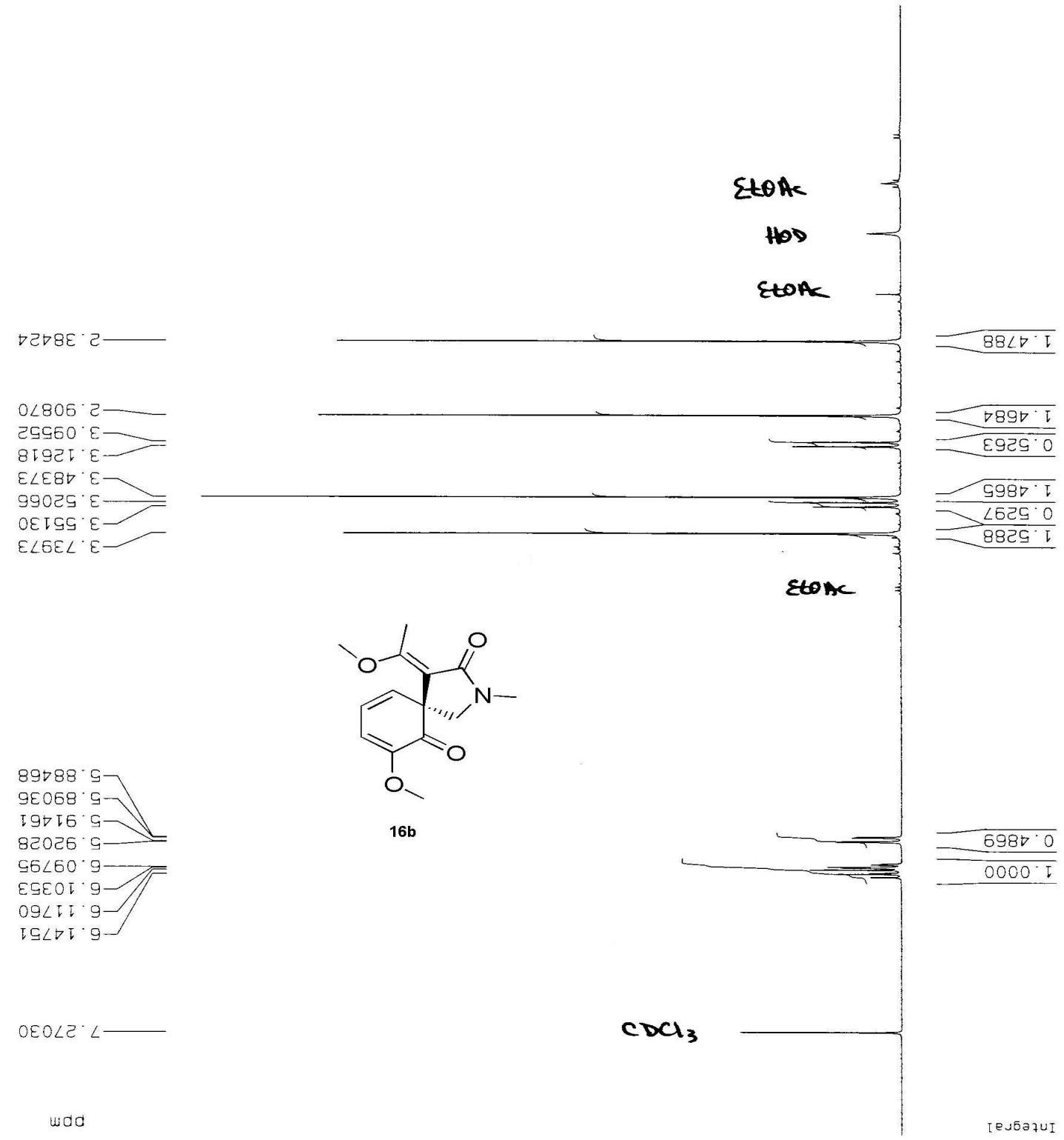




\section{Figure S5}
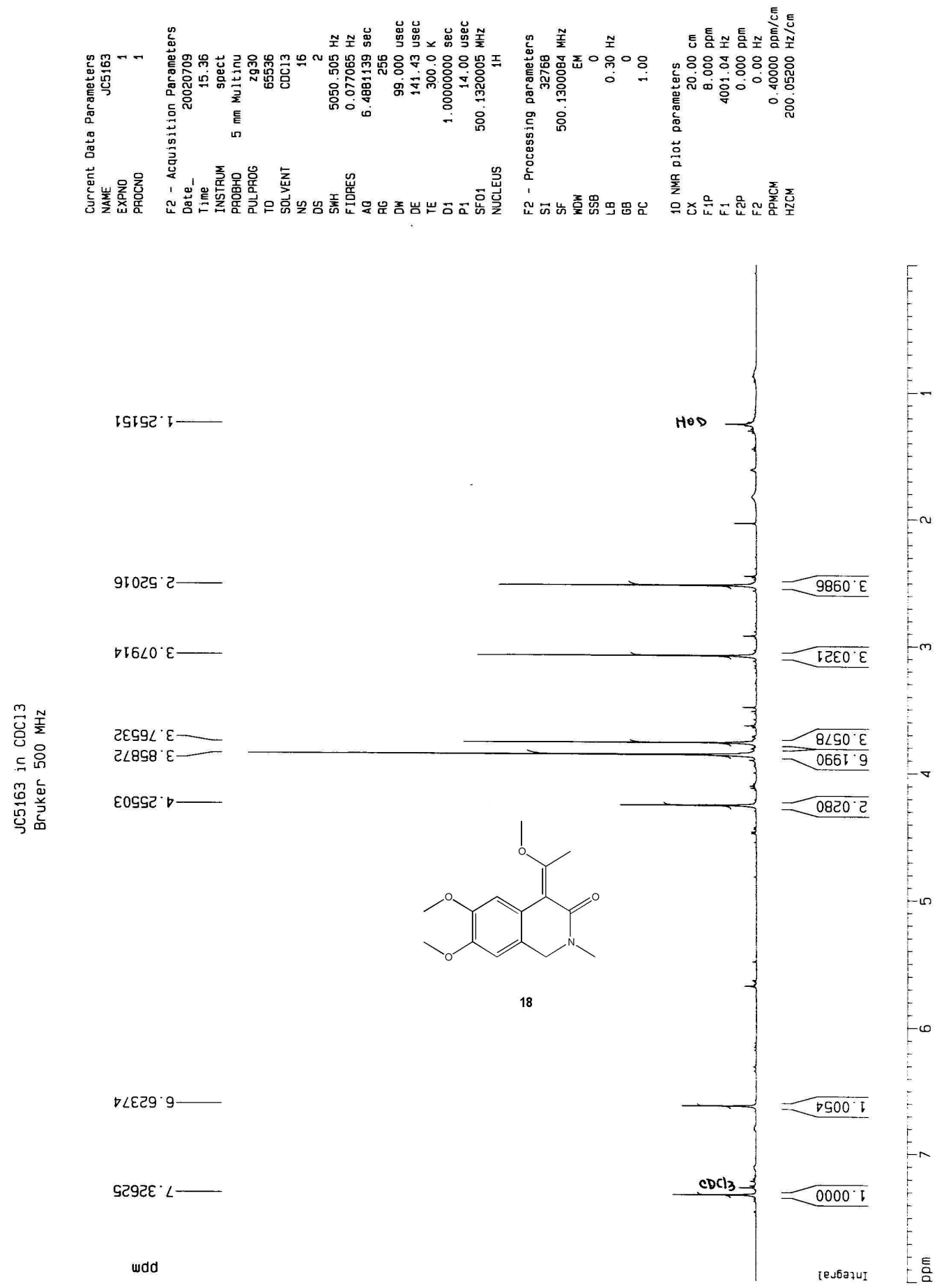


\section{Figure S6}
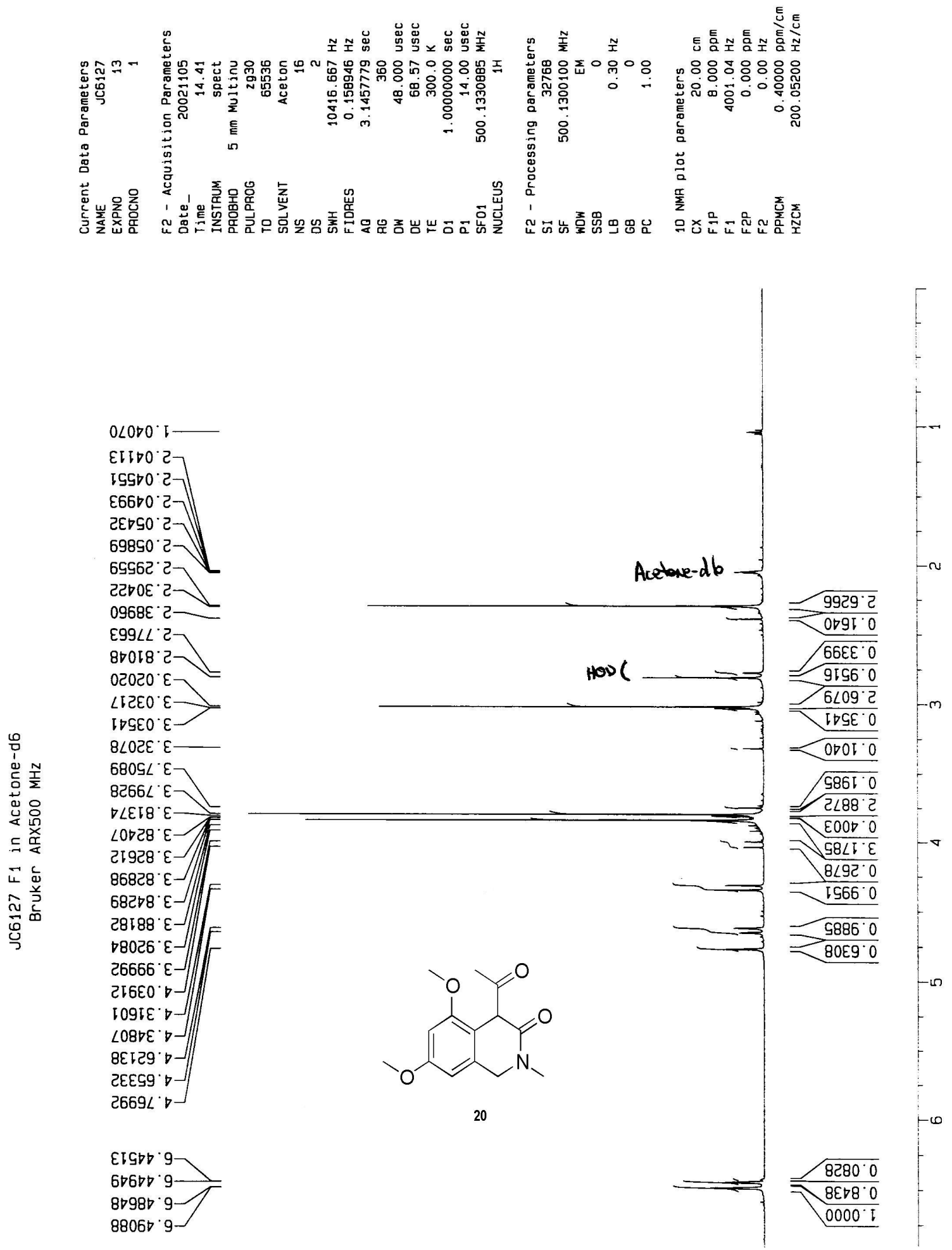


\section{Figure S7}
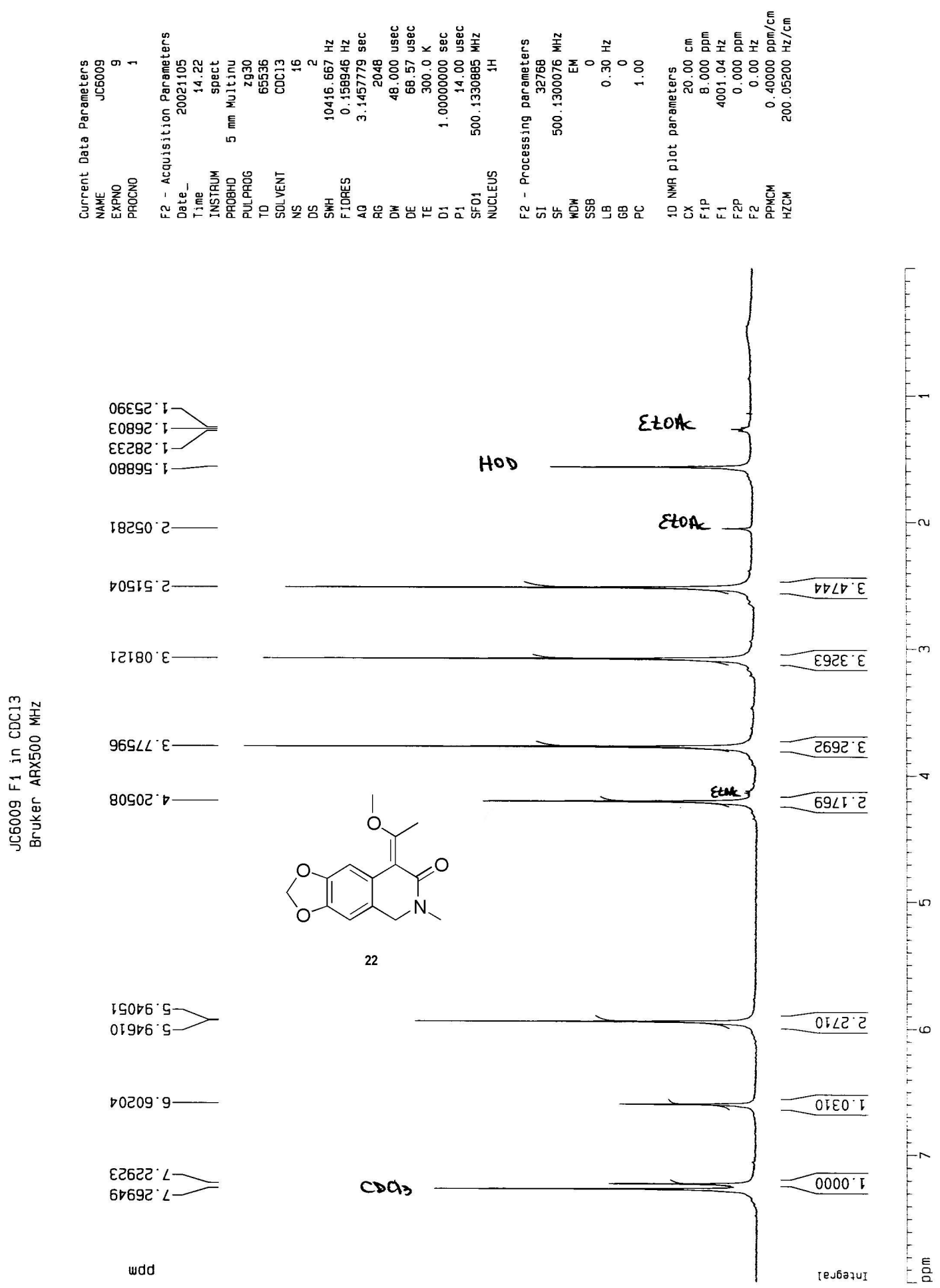


\section{Figure S8}
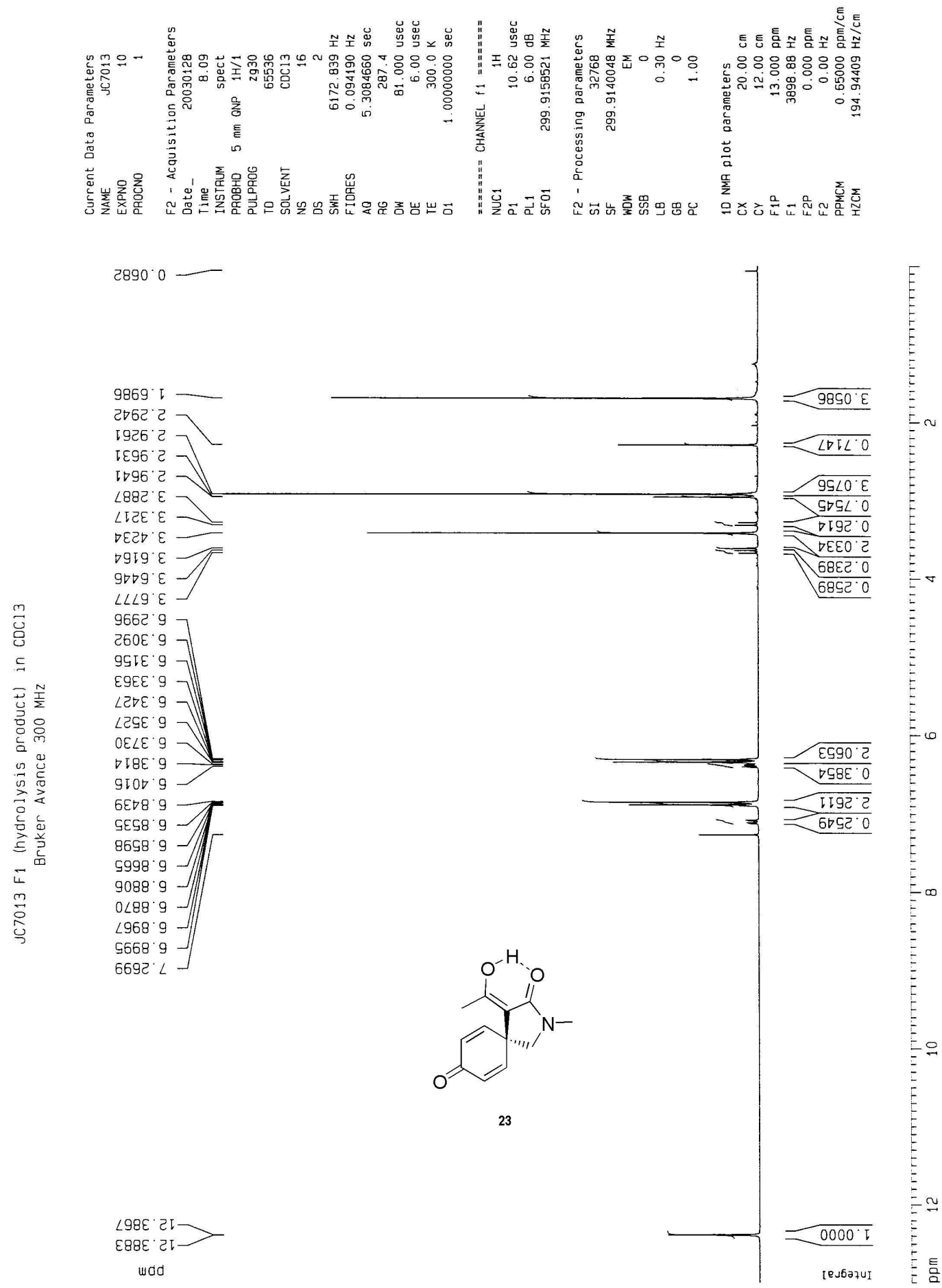


\section{Figure S9}

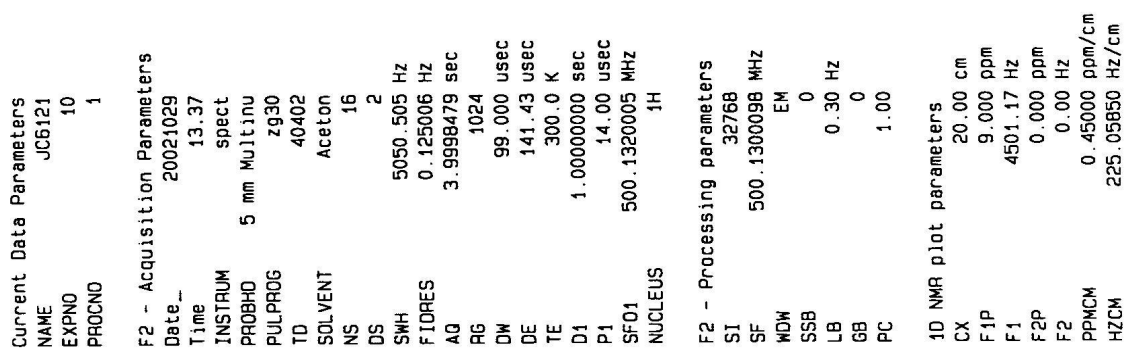

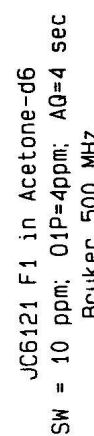

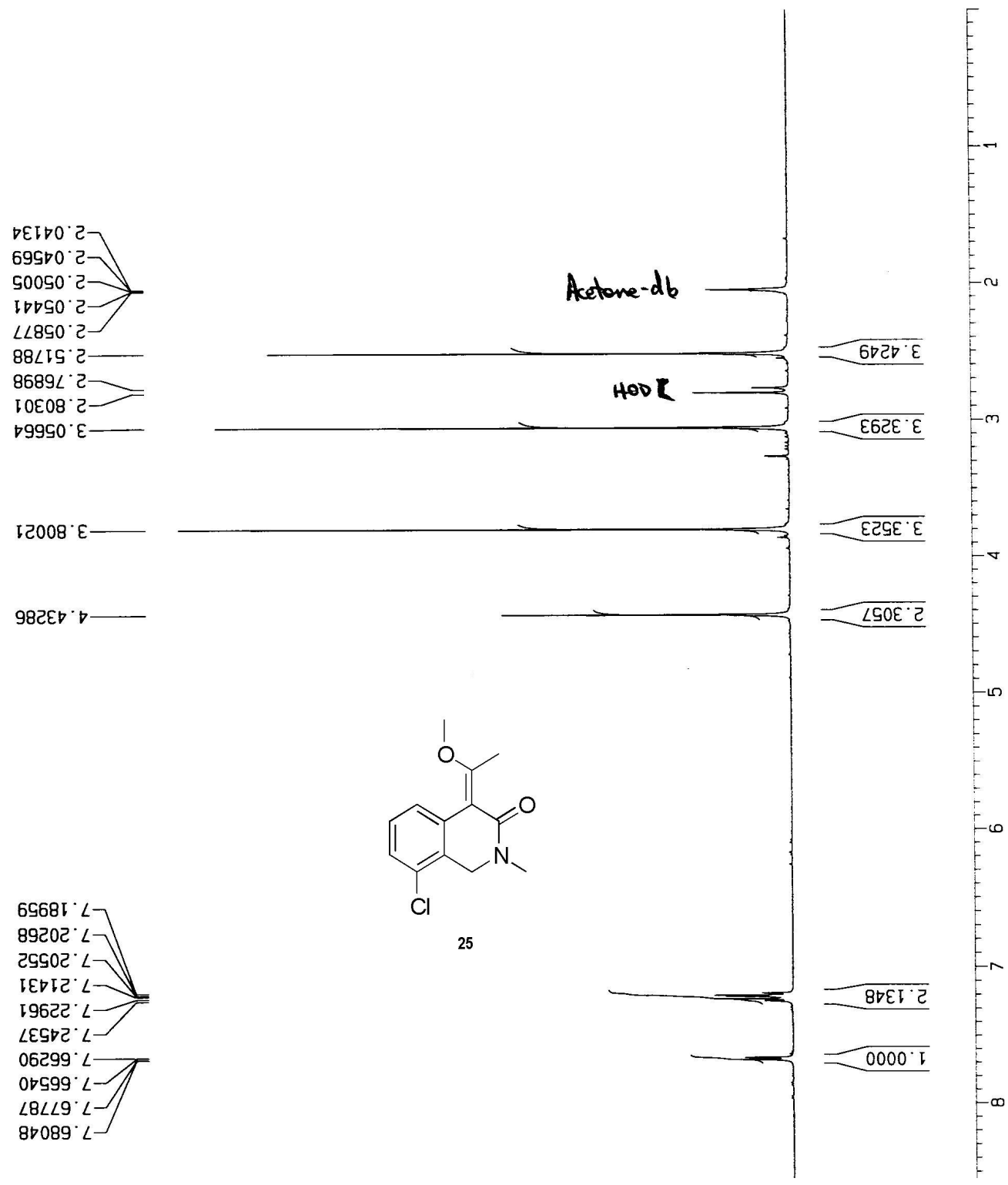




\section{Figure S10}

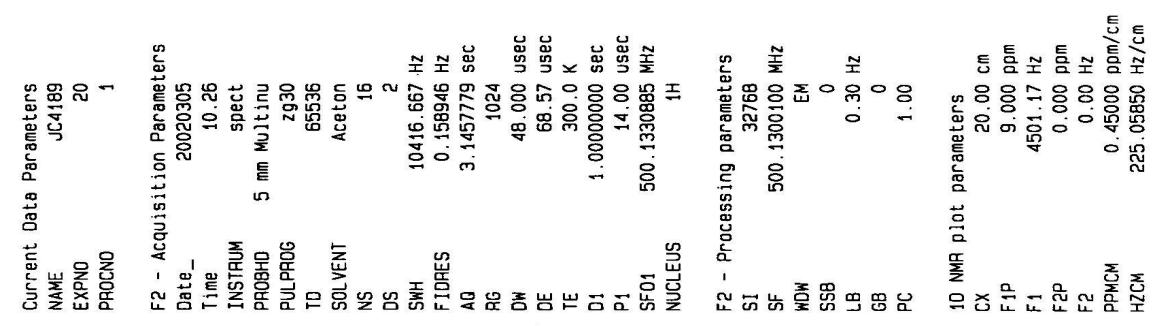

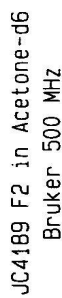
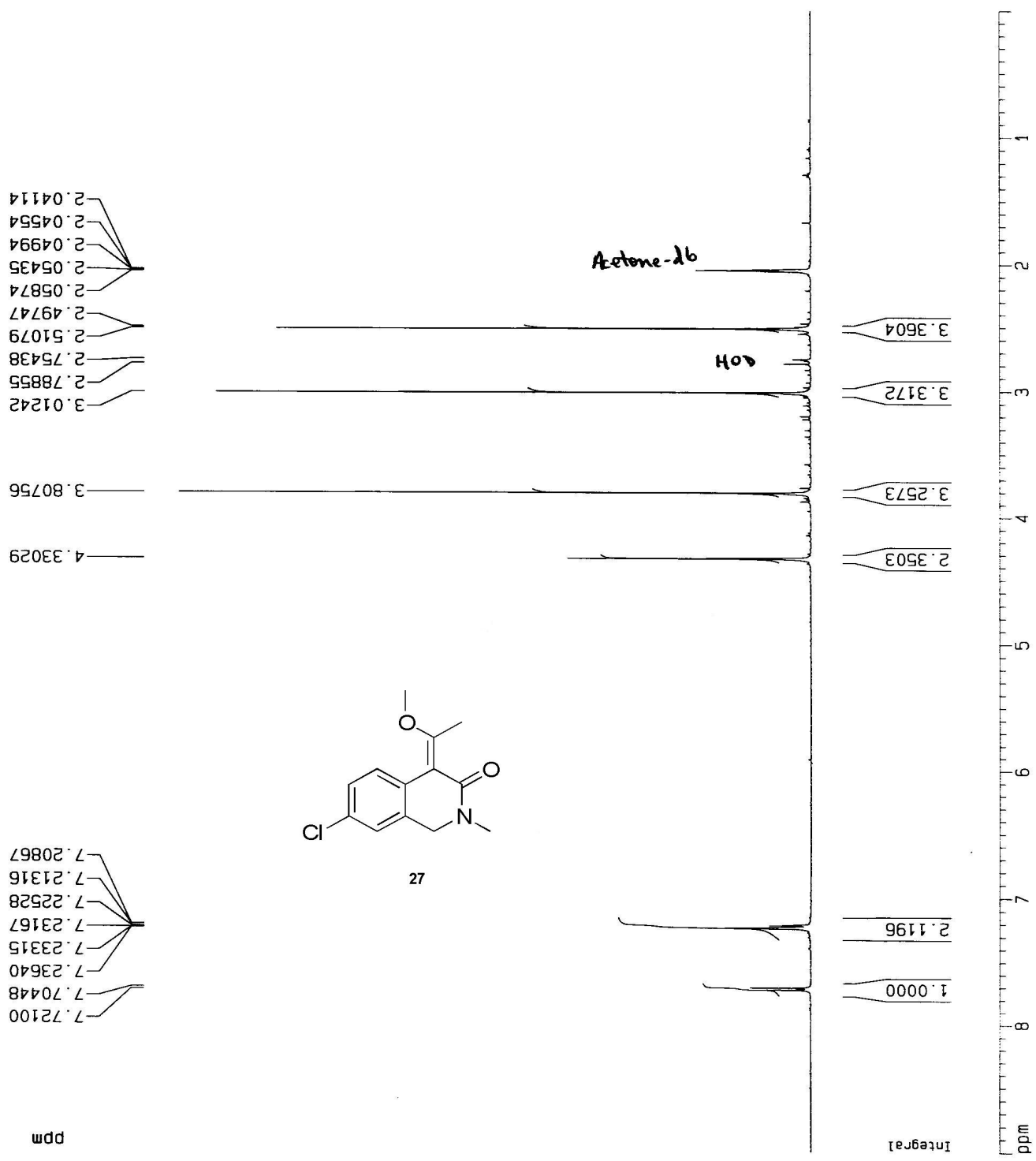

שdd

Actone-d6

$\angle 980 C^{\circ} \angle$ 9IEนट $\angle>$ BटSट2 $\angle>$ $\angle 9 I E C^{\prime} \angle$

SIEEट ' $\angle$

$8 \nabla 00 \angle \circ$

$0012 L \angle-$ 


\section{Figure S11}

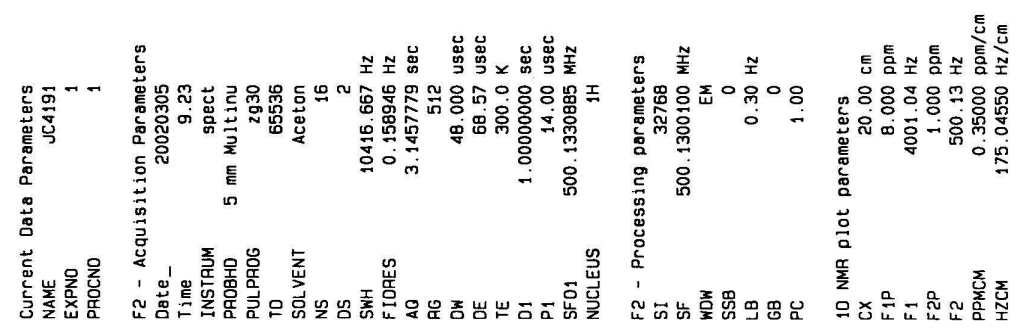

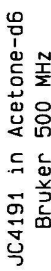
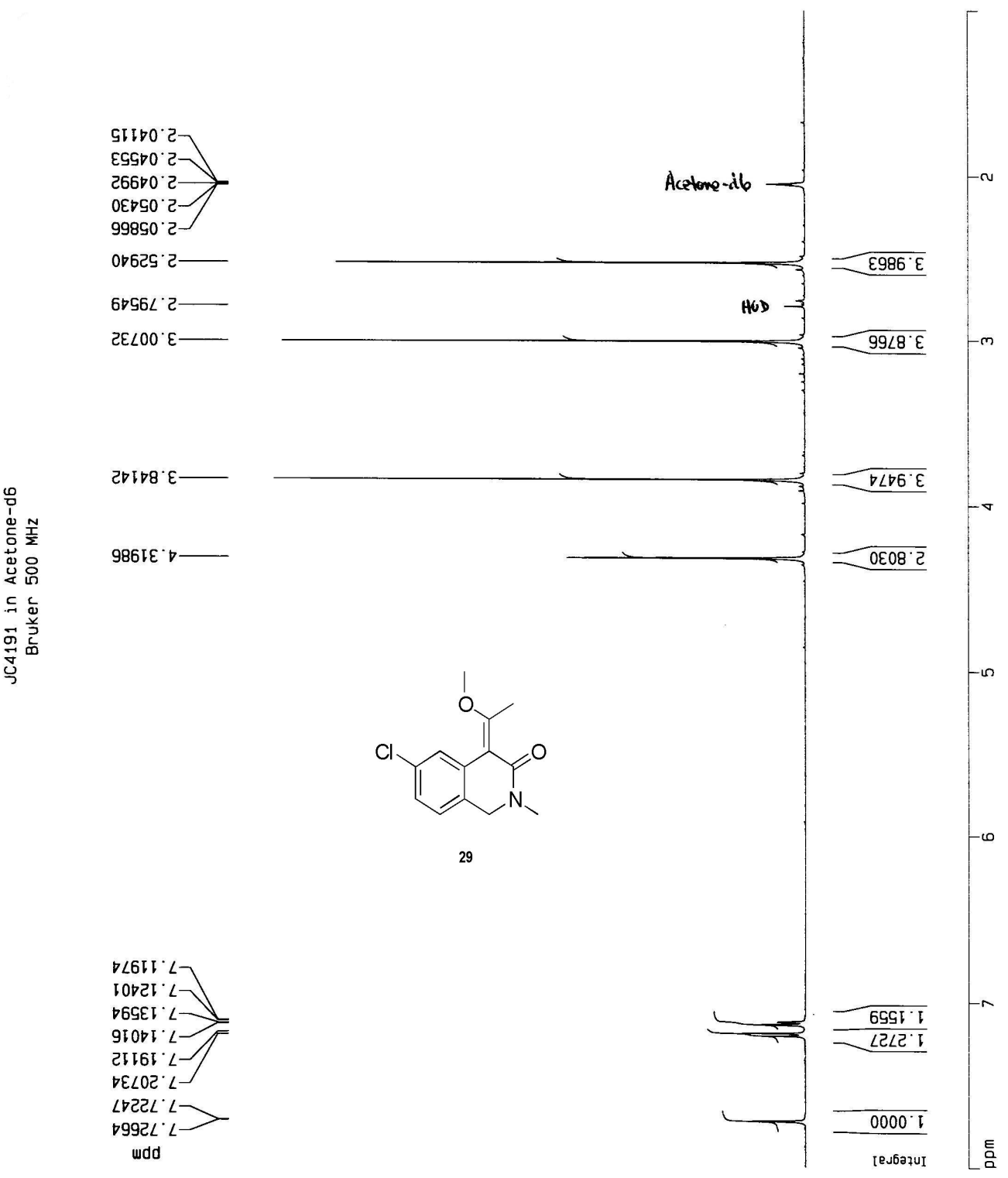


\section{Figure S12}
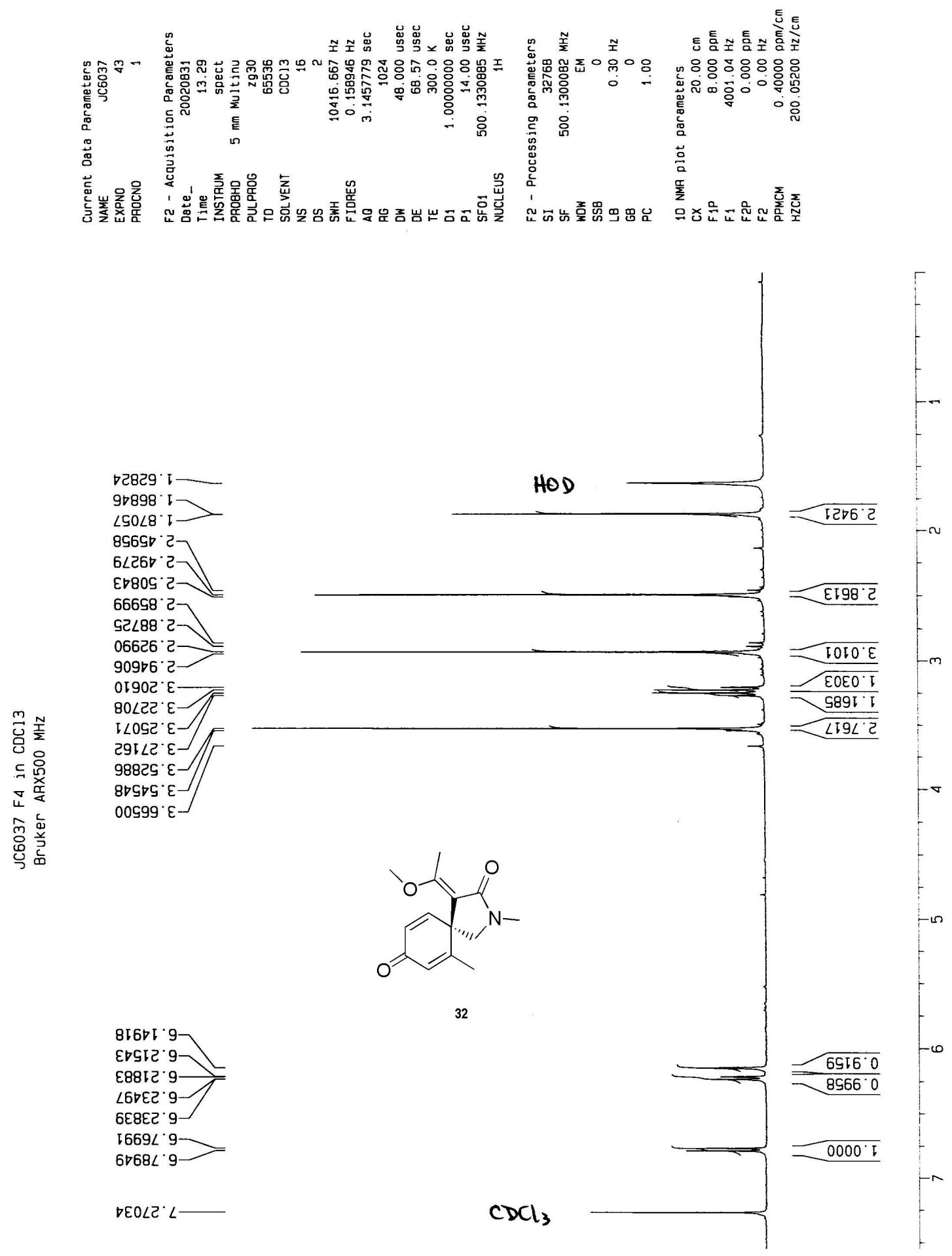


\section{Figure S13}
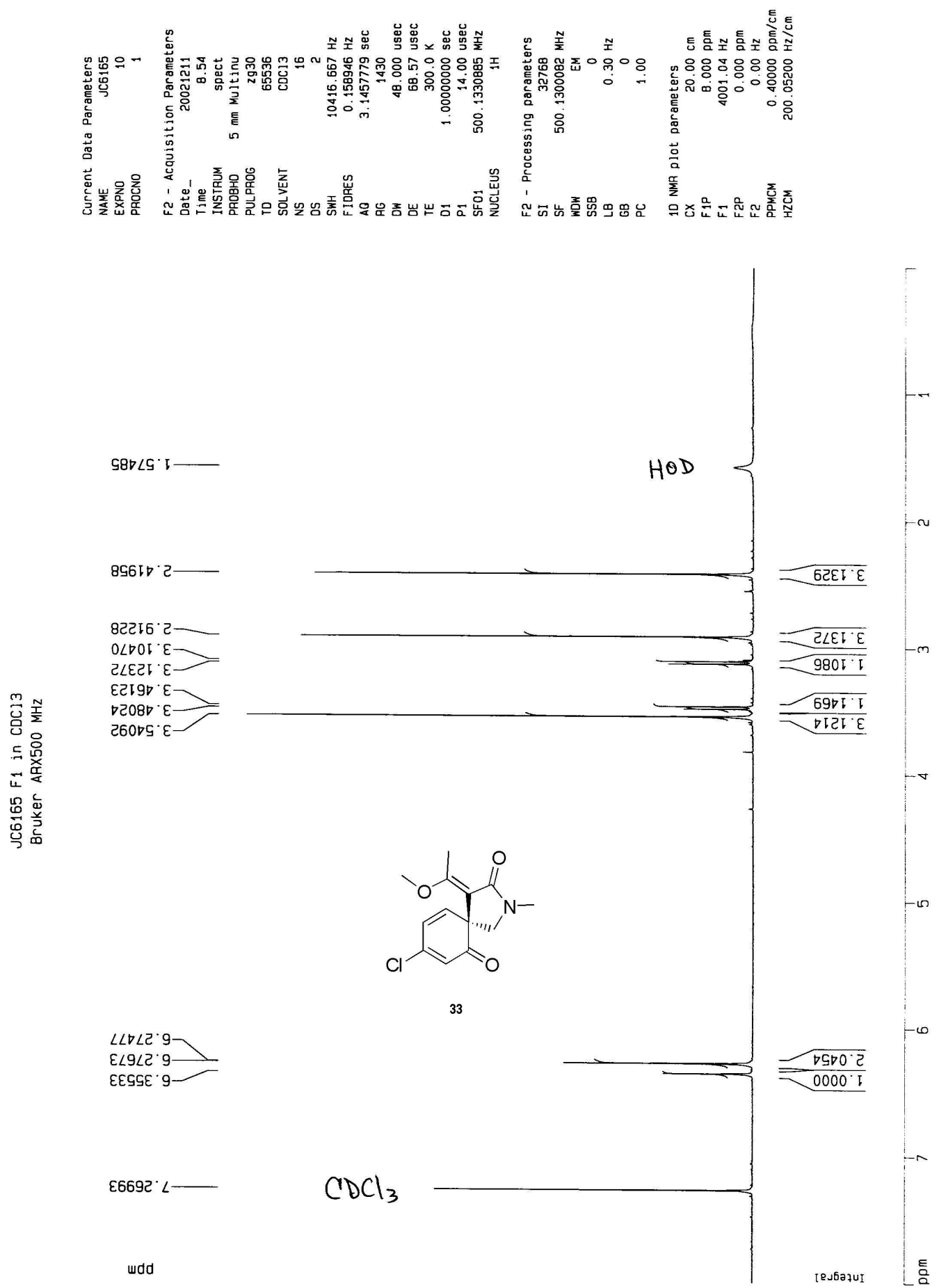


\section{References}

1. Pigge, F. C.; Coniglio, J. J.; Fang, S. Organometallics 2002, 21, 4505.

2. Bruker Analytical X-Ray, Madison, WI, 2001

3. Blessing, R. H. Acta Crystallogr. 1995, A51, 33.

4. Sheldrick, G. M., Bruker Analytical X-Ray Division, Madison, WI, 2001 